\title{
Continued Dispensing: What medications do patients believe should be available?
}

Salem Hasn Abukres, Kreshnik Hoti, Jeffery David Hughes

Background. Continued Dispensing (CD) is a new medication supply method for certain medications in Australia. It aims to prevent treatment interruption as a result of patients' inability to obtain a new valid prescription. The only currently eligible patients for this service are statin and/ oral contraceptive users who: have been using these medications for 6 months or more, have not utilized the CD method during the last 12 months, and cannot obtain an immediate appointment with the prescriber in order to get a new prescription. This study aimed to investigate patients' attitudes towards potential extension and expansion of this medication supply method. Methods. A randomly selected 301 users of these medications from allAustralian States were recruited using Computer Assisted Telephone Interview (CATI). Result. The response rate was $79 \%$. The majority of the participants (73.3\%) did not agree with current restriction on CD utilization frequency. They also supported, to varying degrees, inclusion of all the proposed medications (support ranged from $44.2-78.4 \%$ ). In this regard, participants who suffered from a specific disease did not differ significantly from those without the disease except in case of patients with depression $(p=0.001)$. Conclusions. Participants of this study strongly supported both $C D$ extension and expansion. A future critical review of the current version of $C D$ is highly recommended in order to enhance $C D$ capability to achieve its goals. 


\section{Continued Dispensing: What medications do 3 patients believe should be available?}

4 Salem Hasn Abukres, Kreshnik Hoti, Jeffery David Hughes

5 School of Pharmacy and Curtin Health and Innovation Research Institute, Curtin University, Perth, Western

6 Australia, Australia

7

8

9

10

11 Subject Areas

12 Health Policy, Public Health

13 Keywords

14 Continued Dispensing (CD), Medication supply, Medication access, Chronic diseases, Pharmacists, Australia
15

16

17

18

19

20

21

22

23

24 


\section{Abstract}

26 Background. Continued Dispensing (CD) is a new medication supply method for certain medications in Australia.

27 It aims to prevent treatment interruption as a result of patients' inability to obtain a new valid prescription. The

28 only currently eligible patients for this service are statin and oral contraceptive (OC) users who: have been using

29 these medications for 6 months or more, have not utilized the CD method during the last 12 months, and cannot

30 obtain an immediate appointment with the prescriber in order to get a new prescription. This study aimed to

31 investigate patients' attitudes towards potential extension and expansion of this medication supply method.

32 Methods. A randomly selected 301 users of statin and OC medications from all Australian States were recruited 33 using a Computer Assisted Telephone Interview (CATI).

34 Result. The response rate was $79 \%$. The majority of the participants $(73.3 \%)$ did not agree with current 35 restriction on CD utilization frequency. They also supported, to varying degrees, inclusion of all the proposed 36 additional medications (support ranged from 44.2 - 78.4\%). In this regard, participants who suffered from a specific disease did not differ significantly from those without the disease except in case of patients with depression $(p=0.001)$ who indicated a stronger support level for antidepressant inclusion.

39 Conclusions. Participants of this study strongly supported both CD extension and expansion. A future critical 40 review of the current version of $C D$ is highly recommended in order to enhance CD capability to achieve its 41 goals.

\section{Introduction}

43 Recently in Australia a new method of medications supply, Continued Dispensing (CD), has been implemented 44 to provide patients with a more convenient way to obtain their medications. Patients who run out of statins or oral contraceptives (OCs) are no longer required to present a valid prescription to request these medications under the following conditions: they are unable to obtain an immediate appointment with their doctor, they have been using the medication for more than 6 months, and have not utilized the CD method during the last 12 months.(5th Community Pharmacy Agreement 2013) This system was originally proposed to minimize the risk of patients running out of their medication between doctors' visits. (Bessell et al. 2005)

Medications in Australia are available as: Prescription only medications (Schedule 4, and Schedule 8 for controlled drugs), and non-prescription medications, which include: Pharmacist Only (Schedule3) which can be provided by a pharmacist, Pharmacy Only (Schedule 2) which can be provided by other pharmacy staff under pharmacists' supervision, and other non-scheduled over the counter medications (OTC) which are available for general sale. Statins and OCs (except for the emergency contraceptive pill) are S4 medications, (i.e. a prescription is required for dispensing). However, pharmacists are authorized to dispense these and other types of prescription only medications without a prescription under certain circumstances. For example, as an emergency supply if the patient is a regular user of the requested medication, has no access to the medication 
and lacks a prescription for any valid reason, e.g. traveling or out of date prescription. In such circumstances, the pharmacist may offer a three day supply.(Bessell et al. 2005) Another method to dispense statins or OCs without a prescription is according to the recently introduced CD. According to this method pharmacists can dispense one additional supply (i.e. one standard pack) of the medicine, which is generally enough for one month. Previous literature data suggested that CD eligible patients strongly supported this method.(Abukres ,Hoti \& Hughes 2014) Furthermore, Owing Prescription system is used to continue the supply of medications if obtaining an appointment with the prescriber is not practical. However, this system requires the pharmacist to contact the prescriber before dispensing for approval and the prescriber must provide a written prescription within seven days.(Bessell et al. 2005; Hoti ,Hughes \& Sunderland 2012)

The current $C D$ method may provide limited benefit to chronic disease sufferers as they are often on multiple medications.(Hughes 2005) Consequently, they may present to a pharmacy requesting a statin or an OC, as well as other medications which are currently not eligible for supply under the CD model. In these situations, conducting CD may confuse the patient who can, for instance, obtain their statin without a prescription but cannot obtain their antihypertensive medication. In this situation, CD may not be an appropriate service to offer.

The above suggests that the list of $C D$ eligible medications may need to be expanded to cover a wider range of common diseases, as is the case with pharmacist supplementary prescribing models in other countries.

Pharmacist supplementary prescribing is a partnership between doctors and pharmacists where doctors retain their diagnostic role.(Hoti ,Hughes \& Sunderland 2011a) Patients who experienced supplementary prescribing have shown their support for pharmacists to prescribe a variety of medications such as, but not limited to, medications to treat diabetes, epilepsy, cancer, cardiovascular, respiratory, renal, skin, gastrointestinal, thyroid and blood coagulation diseases.(Lloyd ,Parsons \& Hughes 2010) Furthermore, pharmacist interventions with treatment of chronic diseases have been proven to be effective.(Fikri-Benbrahim et al. 2013; George et al. 2010; Smith et al. 2010) Therefore, applying more responsibility to pharmacists through an expanded version of CD may assist in achieving CD's goals; i.e. a more convenient way for patients to obtain their medication in a timely manner, prevent treatment interruptions, utilize pharmacists' skills and decrease overload on doctors. The Australian Medical Association (AMA) has described the current (limited) CD as unsafe and inappropriate.(Australian Medical Association 2012) This was in contrast with results of a survey of statin and $(\mathrm{OC})$ users, where the majority of the respondents did not perceive CD would pose any risks.(Abukres ,Hoti \& Hughes 2014) They also trusted their pharmacists would conduct CD only when it was safe to do so and that their pharmacist would refer them to their doctor when needed. Furthermore, they thought pharmacists are more easily accessible than doctors; that CD would save their and their doctor' time, and it would help them to not miss any doses of their medications.(Abukres ,Hoti \& Hughes 2014) It is worth mentioning that patients surveyed had no personal experience with $C D$ as the study was conducted before the actual implementation of CD in Australia, so the results represent participates' perceptions rather than their actual experience. Moreover, patients are not necessarily qualified to identify precisely when it's safe or not to obtain a medication without a prescription. 
Another limitation of the $C D$ is its restriction to be conducted only once in any 12 month period. This timeframe has been proposed to prevent patients avoiding doctors' visits.(Bessell et al. 2005) It may lead, however, to treatment interruptions in two ways; if the additional supply is not enough until the next available appointment with the doctor,(Grudzen et al. 2011; Viberg et al. 2013) and/or if the patient runs out of valid prescriptions for their medication more than once in a 12 month period. This may occur as a result of 6 months coverage of chronic medication prescriptions.(Britt 2012)

In this study we sought to explore patients' attitudes towards expansion of CD to include a broader range of medications and hence increasing the access to the service. The study was conducted before the actual implementation of CD on September 1, 2013. In doing so we were able to assess respondents' attitudes before they had experienced the service, and hence without any bias of personal experience.

\section{Methods}

\section{Study design and sampling}

A more detailed methodology of this study has been reported elsewhere.(Abukres, Hoti \& Hughes 2014) Computer-assisted telephone interviewing (CATI) was used. A stratified sample was used. The target sample size was at least 150 statin and 150 OC users, which allowed for a prevalence estimation of $\pm 5 \%$. A telephone number list of a total of 25,000 records was randomly generated based on a broad breakdown of the Australian population by state and territory as outlined in the June 2013 Australian Bureau of Statistics data (i.e. New South Wales 32\%, Victoria 25\%, Queensland $20 \%$, South Australia $7 \%$, Western Australia 11\%, Australian Capital Territory $2 \%$, Tasmania $2 \%$ and Northern Territory 1\%).(Australian Bureau of Statistics 2013)The eligible candidates were users of statins and/or oral contraceptives who were $\geq 18$ years old, spoke English, and used landline phones. Participants who used both medications were interviewed as OC users. Participants were considered consented if they agreed to the question: Would you like to participate? They were told that the interview would take up 15 minutes and they could withdraw at any time. They were called once and if the call timing was not convenient for the interviewee they were called again when it was convenient. Participation was voluntarily and no incentives were used. Ethics approval for the study was obtained from The Human Research Ethics Committee of Curtin University (Approval number: PH-06-13).(Abukres ,Hoti \& Hughes 2014)

\section{Questionnaire design}

The participants were interviewed using a questionnaire consisting of 38 closed ended questions, with the option 'other: please specify' for some questions. The responses were entered into a database verbatim. The questionnaire was developed through a literature review, and experience from a previous study.(Hoti ,Hughes \& Sunderland 2011b) The main focus of the questionnaire was to identify areas of CD extension and expansion, particularly what other medications may potentially be included into the CD system. The challenge was to 
carefully select potential medication classes and medical terms that were easily understandable by the general population. The study tool was assessed for face and content validity by staff members within the pharmacy practice group at Curtin University and by the telemarketing company group CDM Direct Communication Services. The final questionnaire (abridged version Appendix 1) was used by the CDM's trained staff (thus minimizing bias) to collect data during July 2013.(Abukres ,Hoti \& Hughes 2014)

\section{Statistical analysis}

133 The Statistical Package for the Social Sciences (SPSS ${ }^{\text {TM }}$ ) version 22 was used for statistical analysis. In this

134 regard, frequency distribution analysis and Chi square test were employed to assess associations between variables. Answers were collected on a 6 point Likert scale (where $1=$ Strongly disagree, $2=$ Disagree, $3=$ Neutral, $4 .=$ Agree, $5=$ Strongly agree, and $6=$ Prefer not to disclose). For the analysis purposes, the scale was trichotomized as follows: Agreed, which included options 4 and 5, and Disagreed, which included options 1 and 2, and Neutral which included option 3.

\section{Results}

\section{Sample demographic characteristics and response rate}

141 Some of these results have previously reported elsewhere.(Abukres, Hoti \& Hughes 2014) There were 10479 attempts to make phone calls. However, there were a large number of failed calls (7019) due to various reasons, including: number disconnected, answer phones, answering machines, no answer or call busy. This resulted in 3460 successfully contacted individuals. Among these 2,443 individuals were screened out because of ineligibility ( $n=2,146$ who were either under 18 years or not taking a statin or OC, and 297 respondents were deemed unable of completing the survey because of language or hearing difficulties). In addition, there were 716 outright refusals, these were the most problematic because they refused to participate at an early stage before it was clear if they were eligible or not. Since the outright refusals were likely to have a mixed eligibility, the 716 outright refusals were considered to have the same proportion of eligible individuals as the screened sample (i.e. $11 \%$ which was calculated by dividing 301 respondents by 2744). This resulted in an estimated 380 total eligible candidates, giving a response rate of $79 \%$ (301 respondents of the total 380 eligible individuals). However, if all the outright refusals were eligible, which is highly improbable, the response rate could have been as low as $30 \%$. Irrespective of the response rate the targeted sample size was successfully obtained.

154 The respondents were made up of 151 statin and 150 OC users, with the sample consisting of a lower number 155 of male participants compared to females ( $20 \%$ vs $80 \%$, respectively). Their ages were distributed almost

156 equally into those 60 years or younger and those older than 60 years. The participants were recruited from all 157 the Australian states and territories however the state of New South Wales donated almost half of the 158 participants. Only $16 \%$ of the participants were from rural areas.(Abukres ,Hoti \& Hughes 2014)

\section{Participants`attitudes towards expansion of CD}


A. CD extension: Increased access to CD

Participants were asked how many times they thought CD should be allowed within a 12 month period. The majority of participants $(73.3 \%$; $n=222)$ disagreed with the current CD limitation, and selected more than one CD within a 12 month period. Among those who wanted more CD; $16.3 \%$ selected 'twice a year', 5.9\% selected 'three times a year', and $51.1 \%$ of the participants selected 'any time my repeats run out and I am not able to get an appointment with my doctor'.

B. CD expansion: Addition of more medications to the current $C D$ list Participants were asked about their thoughts (i.e. agreement levels) on expanding the current list of CD eligible medications. Table 1 shows the proportion of participants who agreed with inclusion of medications for specific diseases/disorders.

\section{Table 1: Respondents` preferences for medications to be covered under CD}

171 Respondents' support to include particular medications was affected by the condition to be treated. For example, $78.4 \%(n=236)$ of the participants agreed to the inclusion of asthma medications, however only $44.2 \%(n=133)$ agreed to the inclusion of antidepressants. The participants' support for the inclusion of different medications can be divided into three levels based on level of agreement. Level 1: which included medications to treat asthma, arthritis, chronic skin problems, indigestion, hypertension, diabetes (oral hypoglycaemics) and chronic bronchitis, where over $60 \%$ of the participants supported their inclusion within the CD provision. Level 2: which included emphysema medications, chronic pain medications, and anticoagulants where more than 50\% (but less than $60 \%$ ) of the participants agreed to their inclusion, and Level 3: included medications for thyroid disorders, glaucoma, anxiety and depression which were supported by less than $50 \%$ (Table 1).

\section{Views of other disease suffers}

181 More than one third of participants suffered from other chronic diseases $(38.8 \% ; n=116)$. The most prevalent co-morbidities were hypertension, type 2 diabetes mellitus, arthritis, depression, asthma, ingestion, and thromboembolic disorders requiring anticoagulation. Table 2 compares the views of participants with these particular diseases with those without. Generally, in all diseases except type 2 diabetes and indigestion, the proportion of disease suffers who agreed with their medication's inclusion into the CD provisions was higher than the proportion of the total study cohort. However, the only statistically significant difference was between participants supporting inclusion of antidepressants, where participants with depression supported inclusion of these medications more than participants without this disorder $(92.8$ vs $41.8 \%, p=0.001)$. medications in CD

\section{Discussion}

To the best of our knowledge, this is the first study to explore the views of statin and OC users in regards to potential extension and expansion of the CD system in Australia. More specifically, their support to increase the 
194 maximum number of times that CD can be utilized within a 12 month period (i.e. CD extension) and expansion of

195 the range of medications allowed to be dispensed under CD (i.e. CD expansion). Regarding CD extension, the

196 majority of the participants disagreed with the current restriction of $C D$ to once in every a 12 month period, and

197 preferred the option of using it more frequently. Interestingly, more than half of the participants wanted CD to be

198 available until it was possible for them to see their doctor. This may indicate that patients required more flexibility

199 to avoid unnecessary treatment interruption if, for any reason, an appointment with their doctor could not be

200 achieved. Previous studies have reported that patients have difficulty in seeing their regular doctor without a

201 prescheduled appointment.(Gallagher et al. 2001; Garth et al. 2014) Furthermore, it has been reported that

202 patients often do not organize appointments in advance or failed to attend appointments.(Minty \& Anderson

203 2004)

204 On the second question regarding expansion of the medications available through CD, participants generally

205 supported inclusion of more medication classes. However, this support was influenced by the use of those

206 medications. In this regard, the lowest level of support was for medications for the treatment of depression and

207 the highest support was for asthma medications. This profound support for the inclusion of medications to treat a

208 broad range of diseases/disorders may be related to patients' confidence in their self-management and the

209 ability to judge the severity of these diseases. Additionally this may be related to their confidence that

210 pharmacists can provide monitoring for diseases such as diabetes and hypertension. In a previous study,

211 (Wakefield et al. 2000) patients provided reasons for preferring to buy short acting beta agonists (SABAs)

212 without a prescription or with repeats of a previously issued prescription rather than visiting their doctor and

213 obtaining a new prescription after a clinical examination. These reasons included their perception of the

214 worthlessness of visiting their doctor just to obtain a new prescription, their perceptions of medication not

215 requiring such visits and their long experience with the disease, making them feel that they were able to manage

216 and control asthma without the need to see a doctor. This is despite evidence by Braido that "self-reported

217 symptoms poorly correlate with pulmonary function measures".(Braido 2013) Another study reported that

218 obtaining SABAs without a prescription did not lead to poorer asthma control; instead it supported the claim that

219 OTC availability of these medications benefits patients with asthma .(Douglass et al. 2012). Moreover, the

220 availability of some medications to treat asthma such as (SABAs) as a Pharmacist Only medications in Australia,

221 that do not require a prescription, may have increased participants' confidence to obtain more asthma

222 medications without a valid prescription. On the other hand, other studies reported that OTC asthma medicines

223 have resulted in under-treatment and less consultation with doctors. Further, assessment and counselling

224 provided by pharmacists or other pharmacy staff has been reported to be less than optimal.(Schneider et al.

225 2009) However, this inadequate counseling may have resulted from unwillingness of patients with long term

226 chronic diseases to discuss with healthcare professionals what they believed they already know. This

227 controversy about effectiveness and benefit of dispensing asthma medications without a doctors' review raises

228 the need to ensure that optimal patient outcomes are being achieved through appropriate monitoring. This

229 suggests that down scheduling of Prescription Only Medication to Pharmacist Only Medication provides better

230 access to those medications.(Gauld et al. 2014). However, appropriate patient supervision is essential, as is 
231 referral to the doctor whenever deemed necessary.(Abukres ,Hoti \& Hughes 2014)

232 Disease sufferers were more likely to support inclusion of their medications into CD with the exception of 233 patients with diabetes mellitus and indigestion. This is probably because low sample size of patients suffering 234 from ingestion. Interestingly, more than $92 \%$ of patients with depression supported inclusion of antidepressants 235 in $C D$, even though the overall support for the inclusion of medication for depression was the lowest. The exact 236 reason for the difference in support for the inclusion of antidepressants is unclear. However, it may reflect a 237 poorer level of mental health literacy amongst the general population without depression, in which diseases like 238 depression still have a social stigma. The lack of support for the availability of anxiolytics may also be explained 239 in the same way, although the potential for abuse of these medications may also be another explanation. On the 240 other side, the fact that most antidepressants are labelled with warnings "do not to be stopped abruptly" may 241 explain why patients with depression were more supportive than the overall participants. In addition, patients on 242 long-term treatment for depression may not see the need for another visit to the doctor, especially if they do not 243 perceive that they receive any new information during their routine appointments.(Gask et al. 2003) The later 244 may apply to chronic diseases in general, where patients after several years on the same medication may 245 accept that nothing will be change and see their doctor's appointments as adding little value to their 246 management.

247 Participants' support to include additional medications under the CD provisions is consistent with practices in 248 Canada, where pharmacists in some provinces are permitted to undertake short-term dispensing to allow 249 patients to avoid interruption of their continuing therapies.(Law et al. 2012) It is also consistent with the overall 250 trend to expand pharmacists' roles through rescheduling more prescription only medicines to non-prescription 251 status that requires additional pharmacist's intervention (i.e. Pharmacist Only Medications). This includes 252 medications to treat asthma, hypertension and hyperlipidemia.(American Society of Health-System

253 Pharmacists(ASHP) 2012; Gabay 2013; Gauld et al. 2014) Conversely, doctors have expressed their concerns 254 about safety and appropriateness of $C D$,(Daniels 2012) as well as any further reclassifying of prescription only to 255 non-prescription status.(Calabretto 2012)

256 Limitations of this study have been reported in detail previously. (Abukres, Hoti \& Hughes 2014) These include 257 the respondents' distribution, with almost half of the participants being from one state (i.e. New South Wales); 258 the exclusion of those who were under 18 years old, did not speak English, did not use landline phones or were 259 not available at the time of calling. In regard to this section of the study, participants' support to include more 260 medications in the CD model was not based solely on their personal experience, as no participant had all the 261 listed diseases/disorders. In addition, the numbers of participants who suffered from other diseases were low; 262 therefore their support to include their medications may not be generalizable. However, participants' responses 263 may reflect, amongst other things, their general awareness of the disease/disorder, the experience of a friend or 264 relative and/or being a health care professional. The fact that the participants were all either statin or OC users, 265 may bias the results as they may have different views from the general public or patients with the specific 266 diseases, which may limit generalizability of the results of the current study. However, there are factors that 
contribute towards the study strengths, such as being the first about patients' views on the current CD system it may provide insights into how the system be extended and expanded in the future.

269 Future research should explore specific diseases in relation to $C D$, including clinical and economic implications. 270 Further, it is important that research undertaken to assess whether patients' expressed desire to expand and extend $C D$ is in their best health and economic interests. Given the limitations of the current CD method, other medication supply models to patients in cases where there is lack of valid prescription should also be explored.

\section{Conclusions}

274 Current restrictions on CD may limit its capacity to serve its goals, as suggested by this study with participants

275 highly supporting a more flexible and broader CD system. The currently eligible utilizers of the CD system seem

276 to prefer inclusion of additional medications and more opportunity to use CD at any time they cannot see their 277 doctor. These findings suggest that ongoing review of CD is essential and changes which do not compromise 278 patient safety or allow the abuse of CD would be welcomed by patients.

\section{Additional Information}

\section{Competing Interests}

281 Authors declare no relevant competing interests related to this work.

\section{Funding}

283 There was no funding received for this study.

\section{References}

285

286 5th Community Pharmacy Agreement. 2013. The continued dispensing of pharmaceutical benefits scheme medicines in defined circumstances (continued dispensing) initiative. Available at http://www.5cpa.com.au/docs/librariesprovider5/public-documents/initiatives-and-programs/continueddispensing/continued-dispensing-fact-sheet.pdf?sfvrsn=2 (accessed Februrary 10, 2014).

Abukres SH, Hoti K, and Hughes JD. 2014. Patient attitudes towards a new role for pharmacists: Continued dispensing. Patient Preference and Adherence 8:1143.

American Society of Health-System Pharmacists(ASHP). 2012. ASHP highlights the importance of pharmacists in nonprescription. Available at http://www.ashp.org/DocLibrary/Advocacy/Nonprescription-use-ofprescription-drugs.aspx, (accessed June 10, 2014).

Australian Bureau of Statistics. 2013. Australian demographic statistics. Available at http://www.abs.gov.au/AUSSTATS/abs@.nsf/allprimarymainfeatures/61D19DFBD033B48FCA257CA70 OODCB16?opendocument (accessed June 30, 2013).

Australian Medical Association. 2012. Guidelines for continued dispensing of eligible prescribed medicines by pharmacists. Available at http://ama.com.au/node/7453 (accessed April 18, 2012).

Bessell T, Marriott J, Emmerton L, and Nissen L. 2005. Improving Australians' access to prescription medicines: Development of pharmacy practice models. Available at http://www.guild.org.au/docs/defaultsource/public-documents/services-and-programs/research-and-development/Third-Agreement- $R$-andD/2003-017/final-report.pdf?sfvrsn=0 (accessed March 6, 2014).

Braido F. 2013. Failure in asthma control: Reasons and consequences. Scientifica (Cairo) 2013:549252. 
304

305

306

307

308

309

310

311

312

313

314

315

316

317

318

319

320

321

322

323

324

325

326

327

328

329

330

331

332

333

334

335

336

337

338

339

340

341

342

343

344

345

346

347

348

349

350

351

352

353

354

355

356

357

Britt H. 2012. General practice activity in australia 2011-12: Beach, bettering the evaluation and care of health: Sydney University Press.

Calabretto H. 2012. Australia: Organized physician opposition to nonprescription status. In: Foster A M WLe, ed. Emergency contraception - the story of a global reproductive health technology. New York City: Palgrave Macmillan Limited, 207-220.

Daniels A. 2012. Be on it or be under it-the chronic health care road train is coming. Australian Pharmacist 31:624.

Douglass JA, Goeman DP, McCarthy EA, Sawyer SM, Aroni RA, Stewart K, and Abramson MJ. 2012. Over-thecounter $\beta 2$-agonist purchase versus script: A cross-sectional study. Respiratory Medicine 106:223-229.

Fikri-Benbrahim N, Faus MJ, Martínez-Martínez F, and Sabater-Hernández D. 2013. Impact of a community pharmacists' hypertension-care service on medication adherence. The afenpa study. Research in Social and Administrative Pharmacy 9:797-805.

Gabay M. 2013. Expanding the definition of nonprescription drugs: Potential impact on pharmacists. Hospital Pharmacy 48:198-199.

Gallagher M, Pearson P, Drinkwater C, and Guy J. 2001. Managing patient demand: A qualitative study of appointment making in general practice. British Journal of General Practice 51:280-285.

Garth B, Temple-Smith M, Clark M, Hutton C, Deveny E, Biezen R, and Pirotta M. 2014. 'Your lack of organisation doesn't constitute our emergency'-repeat prescription management in general practice. Australian Family Physician 43:404.

Gask L, Rogers A, Oliver D, May C, and Roland M. 2003. Qualitative study of patients' perceptions of the quality of care for depression in general practice. British Journal of General Practice 53:278-283.

Gauld NJ, Kelly FS, Kurosawa N, Bryant LJ, Emmerton LM, and Buetow SA. 2014. Widening consumer access to medicines through switching medicines to non-prescription: A six country comparison. PloS One 9:107726.

George PP, Molina J, Cheah J, Chan SC, and Lim BP. 2010. The evolving role of the community pharmacist in chronic disease management-a literature review. Annals of the Academy of Medicine, Singapore 39:861-867.

Grudzen CR, Stone SC, Mohanty SA, Asch SM, Lorenz KA, Torres JM, Morrison M, Ortiz JM, and Timmermans S. 2011. "I want to be taking my own last breath": Patients' reflections on illness when presenting to the emergency department at the end of life. Journal of Palliative Medicine 14:293-296.

Hoti K, Hughes J, and Sunderland B. 2011a. An expanded prescribing role for pharmacists-an Australian perspective. The Australasian Medical Journal 4:236.

Hoti K, Hughes J, and Sunderland B. 2011b. Pharmacy clients' attitudes to expanded pharmacist prescribing and the role of agency theory on involved stakeholders. International Journal of Pharmacy Practice 19:5-12.

Hoti K, Hughes J, and Sunderland B. 2012. Medication supply to residential aged care facilities in Western Australia using a centralized medication chart to replace prescriptions. BMC Geriatrics 12:25.

Hughes JD. 2005. Focusing on the patient's concerns. Australian Pharmacist 24 538-539.

Law MR, Ma T, Fisher J, and Sketris IS. 2012. Independent pharmacist prescribing in Canada. Canadian Pharmacists Journal/Revue des Pharmaciens du Canada 145:17-23.

Lloyd F, Parsons C, and Hughes CM. 2010. 'It's showed me the skills that he has': Pharmacists' and mentors' views on pharmacist supplementary prescribing. International Journal of Pharmacy Practice 18:29-36.

Minty B, and Anderson C. 2004. Non-attendance at initial out-patient appointments at a hospital-based child psychiatric clinic. Clinical Child Psychology and Psychiatry 9:403-418.

Schneider CR, Everett AW, Geelhoed E, Kendall PA, and Clifford RM. 2009. Measuring the assessment and counseling provided with the supply of nonprescription asthma reliever medication: A simulated patient study. Annals of Pharmacotherapy 43:1512-1518.

Smith M, Bates DW, Bodenheimer T, and Cleary PD. 2010. Why pharmacists belong in the medical home. Health Affairs 29:906-913.

Viberg N, Forsberg BC, Borowitz M, and Molin R. 2013. International comparisons of waiting times in health care-limitations and prospects. Health Policy 112:53-61.

Wakefield M, Ruffin RE, Wilson DHD, Campbell DA, Chittleborough CR, and Farmer EA. 2000. Over-thecounter purchase of inhaled $\beta 2$ agonists: A qualitative study of asthma patients. Health Promotion Journal of Australia 10:38-42. 
363

364 
Table $\mathbf{1}$ (on next page)

Table 1: Respondents` preferences for medications to be covered under CD

Note: For every question about Disease/Disorder/Medication there were participants who preferred to not 5 disclose 
2 Table 1: Respondents` preferences for medications to be covered under CD

3

\begin{tabular}{|c|c|c|c|}
\hline Disease/Disorder/Medication & Neutral & Disagreed & Agreed \\
\hline & $\mathrm{n}(\%)$ & n (\%) & $\mathrm{n}(\%)$ \\
\hline Asthma & $30(10.0)$ & $31(10.3)$ & $236(78.4)$ \\
\hline Arthritis & $35(11.6)$ & $35(11.6)$ & $227(75.4)$ \\
\hline Chronic skin disorders & $36(12.0)$ & $39(13.0)$ & $222(73.8)$ \\
\hline Indigestion & $38(12.6)$ & $40(13.3)$ & $219(72.8)$ \\
\hline Hypertension & $36(12.0)$ & $48(15.9)$ & $213(70.8)$ \\
\hline Diabetes & $58(19.3)$ & $37(12.3)$ & $202(67.1)$ \\
\hline Chronic bronchitis & $54(17.9)$ & 55 (18.3) & $175(58.1)$ \\
\hline Emphysema & $63(20.9)$ & $59(19.6)$ & $161(53.5)$ \\
\hline Chronic pain & $50(16.6)$ & $86(28.6)$ & $153(51.2)$ \\
\hline Blood clotting & $58(19.3)$ & $85(28.2)$ & $150(49.8)$ \\
\hline Thyroid & $80(26.6)$ & $67(22.3)$ & $150(49.8)$ \\
\hline Glaucoma & $76(25.2)$ & $71(23.6)$ & $145(48.2)$ \\
\hline Anxiety & $54(17.9)$ & $98(32.6)$ & $133(44.2)$ \\
\hline Depression & $51(16.9)$ & $113(37.5)$ & $175(58.1)$ \\
\hline
\end{tabular}

$4 \quad$ Note: For every question about Disease/Disorder/Medication there were 4 participants who preferred to not 5 disclose

6 
Table 2 (on next page)

Table 2: Influence of experience with the disease on respondents' attitudes to the inclusion of particular medications in CD 
2 Table 2: Influence of experience with the disease on respondents' attitudes to the inclusion of particular 3 medications in CD

\begin{tabular}{|llll|}
\hline Disease & $\begin{array}{l}\text { Agreed participants without the } \\
\text { disease } \mathrm{n}(\%)\end{array}$ & $\begin{array}{l}\text { Agreed participants with the } \\
\text { disease } \mathrm{n}(\%)\end{array}$ & P value \\
\hline Hypertension & $180(68.7)$ & $33(84.6)$ & 0.06 \\
Diabetes mellitus & $186(67.4)$ & $16(64.0)$ & 0.91 \\
Arthritis & $222(79.0)$ & $14(70.0)$ & 0.51 \\
Depression & $120(41.8)$ & $13(92.8)$ & 0.001 \\
Asthma & $225(77.8)$ & $11(91.7)$ & 0.23 \\
Indigestion & $214(72.8)$ & $5(71.4)$ & 0.36 \\
Blood clotting & $154(50.8)$ & $4(66.7)$ & \\
\hline
\end{tabular}

4 